\title{
Article \\ Multilevel Analysis of the Nutritional and Health Status among Children and Adolescents in Eastern China
}

\author{
Ting Tian ${ }^{1}$, Yuanyuan Wang ${ }^{1,2}$, Wei Xie ${ }^{1}$, Jingxian Zhang ${ }^{1}$, Yunlong $\mathrm{Ni}^{1}{ }^{1}$, Xianzhen Peng ${ }^{3}$, Guiju Sun ${ }^{2} \mathbb{1}$, \\ Yue Dai ${ }^{1}$ and Yonglin Zhou ${ }^{1, *}$
}

check for

updates

Citation: Tian, T.; Wang, Y.; Xie, W.; Zhang, J.; Ni, Y.; Peng, X.; Sun, G.; Dai, Y.; Zhou, Y. Multilevel Analysis of the Nutritional and Health Status among Children and Adolescents in Eastern China. Nutrients 2022, 14, 758 . https://doi.org/10.3390/ nu14040758

Academic Editor: Zhiyong Zou

Received: 12 January 2022

Accepted: 6 February 2022

Published: 11 February 2022

Publisher's Note: MDPI stays neutral with regard to jurisdictional claims in published maps and institutional affiliations.

Copyright: (C) 2022 by the authors. Licensee MDPI, Basel, Switzerland. This article is an open access article distributed under the terms and conditions of the Creative Commons Attribution (CC BY) license (https:// creativecommons.org/licenses/by/ $4.0 /)$.
1 Institute of Food Safety and Assessment, Jiangsu Provincial Center for Disease Control and Prevention, Nanjing 210009, China; jstt@jscdc.cn (T.T.); wyypro@foxmail.com (Y.W.); jscdcxiewei@sina.com (W.X.); z18252063009@163.com (J.Z.); nyl2008@163.com (Y.N.); 18915999341@163.com (Y.D.)

2 Department of Nutrition and Food Hygiene, School of Public Health, Southeast University, Nanjing 210009, China; gjsun@seu.edu.cn

3 Department of Public Health and Preventive Medicine, Kangda College of Nanjing Medical University, Lianyungang 222000, China; xianzhenpeng@njmu.edu.cn

* Correspondence: jsepipublic@sina.com

\begin{abstract}
We aimed to identify multiple nutritional health problems and the relevant factors among children and adolescents aged 7-17 years. This study was part of the China Nutrition and Health Surveillance of Children and Lactating Mothers in 2016-2017, conducted in Jiangsu Province in eastern China. After sampling, 3025 school-age children and adolescents were enrolled into this study. Demographic information collections and anthropometric measurements were conducted by trained local Center for Disease Control and Prevention (CDC) staff. Venous blood in the amount of $6 \mathrm{~mL}$ was drawn from each participant in the morning and used for testing biochemical and nutritional indicators. Multivariate logistic regression analysis and Poisson regression analysis were used for overnutrition- and undernutrition-related disorders to test relevant personal, parental, and household factors. The prevalence of wasting, overweight, and obesity was $5.5 \%, 14.8 \%$, and $12.7 \%$, respectively. Metabolic syndrome (MetS) was prevalent among $5.1 \%$ of participants. Among the study participants, $29.5 \%$ had hyperuricemia. The overall prevalence of high low-density lipoprotein (LDL) and high total cholesterol (TC) of all participants was $4.8 \%$ and $7.4 \%$, respectively. $0.9 \%$ of the participants had vitamin A deficiency (VAD) and $14.6 \%$ had marginal vitamin A deficiency; $25.1 \%$ had vitamin $\mathrm{D}$ deficiency (VDD) and $54.5 \%$ had inadequate vitamin D levels. Anemia was present in $4.0 \%$ of all participants. The prevalence of zinc deficiency was $4.8 \%$. Demographic characteristics, behavioral characteristics, parents' characteristics, and family characteristics were associated with these multiple malnutrition disorders. The double burdens of malnutrition, which includes overnutrition- and undernutrition-related diseases, were prevalent among the school-age children and adolescents in Jiangsu Province in eastern China. There were various factors related to different nutritional problems. Thus, health education focusing on behavior intervention and nutrition education are necessary in containing nutritional problems among children.
\end{abstract}

Keywords: nutritional status; children and adolescents; malnutrition; overnutrition

\section{Introduction}

Nutrition is essential for children's neurodevelopment and reproductive development and is associated with linear growth [1]. Improving children's nutrition is urgently needed for global development and for reducing the burden of noncommunicable diseases (NCDs) [2]. Nutritional conditions in the early stages of life may have both short-term and longer lasting effects, including an increased risk of NCDs and other chronic diseases [3]. Cardiovascular diseases, which are the most common causes of mortality among adults worldwide, are rooted in childhood, underlying the urgency of identifying and intervening with at-risk children $[4,5]$. 
Further, approximately one-half of all childhood deaths globally are caused directly or indirectly by malnutrition [6]. Various malnutrition diseases, including undernutritionand overnutrition-related disorders, are highly prevalent among children and adolescents in many countries $[7,8]$. For example, vitamin A deficiency (VAD) remains a public health problem in China, where the prevalence of VAD in children was 5.16\% in 2015 [9]. A high prevalence of vitamin D deficiency (VDD) has also been reported in infants, children, and adolescents from diverse countries around the world [10]. The overall prevalence of anemia was $8.8 \%$ among children in Hunan Province in China [11] and 17.49\% among school-age children in Latin America and the Caribbean [12]. Zinc is an essential trace element for growth and development in children, but zinc deficiency is a serious nutritional problem in China. In 2012, the China Nutrition and Health Survey showed a $6.8 \%$ prevalence of zinc deficiency in 3407 school-age children [13].

In addition to undernutrition, overnutrition is a serious problem. The results of five consecutive national surveys from 1995 to 2004, as part of the Chinese National Survey on Students' Constitution and Health, showed that the main growth problems had changed from undernutrition to overnutrition in the past 20 years [14]. The nutrition issues of children and adolescents have become more important in view of the global epidemic of obesity, metabolic syndrome (MetS), and other metabolic risk factors. Worldwide, more than 100 million children are obese [15].

One predictor of future health risk is Metabolic syndrome (MetS), a cluster of cardiovascular risk factors that include abdominal obesity, which is mainly measured by high waist circumference (WC), hypertension, high fasting triglycerides (TG), low high-density lipoprotein (HDL) cholesterol, and high fasting blood glucose (FBG) [16]. The recent prevalence of MetS in American children and adolescents was 9.83\%, according to the results of National Health and Nutrition Examination Survey (NHANES) [17]. Data from the China Health and Nutrition Surveys (CHNS) showed that the prevalence of MetS in Chinese children and adolescents aged 7-18 years was 3.37\% [18]. Meanwhile, recent evidence suggests that hyperuricemia is at a high level in children and adolescents in both China and America, with the prevalence ranging from $10.1 \%$ to $26.02 \%$ [19-21].

With sustained economic development, the living standards of Chinese residents, and especially residents of Jiangsu Province, which is a relatively developed area in the country, have continuously improved. However, China still faces many nutritional problems, such as the coexistence of undernutrition- and overnutrition-related disorders among children and adolescents and the lack of clarity regarding the sociodemographic factors that are related to these nutritional problems. Sociodemographic differences play important roles in nutrition status, especially among children and adolescents [22-24]. To date, there have been few studies conducted in Jiangsu Province to describe comprehensively the nutritional status of school-age children and adolescents. Thus, this study aimed to identify the multiple nutritional health problems among children and adolescents aged 7-17 years in Jiangsu Province in China, and the relevant factors contributing to those problems, based on provincial representative data.

\section{Materials and Methods}

\subsection{Study Participants}

This study was part of the China Nutrition and Health Surveillance of Children and Lactating Mothers in 2016-2017. We selected school-age children and adolescents from 12 survey sites, including urban and rural areas in Jiangsu Province in eastern China. The representative provincial samples for accessing the nutritional and health status of schoolaged participants were randomly selected by multistage stratified cluster random sampling methods. The study design and sampling methods were described by Dongmei Yu et al. in an article introducing the China Nutrition and Health Survey from 1982-2017 [25].

In the first stage of the study, 12 survey sites were randomly picked from the whole province, including two survey sites in large cities, eight sites in medium cities, and two sites in generally rural counties. In the second stage, two townships/subdistricts were 
randomly picked from each survey site. In the third stage, two communities or villages of township/subdistricts were randomly selected. In the last stage, 70 school-age children and adolescents were randomly picked from the selected communities or villages. There were at least 280 children aged 7-17 years, with equal numbers of males and females, selected in each of the survey sites. Replacements were allowed only from the same village/neighborhood and similar households as those of the original participants. The replacement rate had to be less than 35\%. After sampling, 3360 school-aged children and adolescents were enrolled into the study. In the final analysis, 3025 participants were included, after excluding participants who were unable to provide key information, such as sociodemographic characteristics, lab-testing information, or anthropometric results $(n=335)$.

\subsection{Ethics Approval and Consent to Participate}

This study was performed according to the Declaration of Helsinki and approved by the ethical committee of the China Center for Disease Control and Prevention (the China CDC); the corresponding ethical approval number was 201614. All of the participants agreed to take part in this study and signed the required informed consent form.

\subsection{Data Collection and Measurements}

Interviews were based on household and individually structured questionaries provided by the China CDC project group, to collect demographic information and additional information concerning physical activities, screen time, parents, household sizes, and other details. Interviews were conducted by a strictly trained investigator in a face-to-face manner.

Screen time refers to the time using an electronic screen every day. A low level of screen time was defined as $<2 \mathrm{~h}$ and a high level was defined as $\geq 2 \mathrm{~h}$. The assessment of physical activities was based on reaching a level of coarsened breathing or a heart rate rise for more than $30 \mathrm{~min}$. An exercise level of 0-3 days per week was defined as a low physical activity level; an exercise level for more than 4 days per week was defined as a high physical activity level.

Anthropometric measurements including height, weight, WC, and blood pressure were conducted by trained local CDC staff. All equipment used in the measurements were selected according to the guidance of the national project group. Height and weight were measured without shoes or coats. A stadiometer (TZG, Nantong, China) was used for height measurement, with an accuracy to $0.1 \mathrm{~cm}$. The equipment used for weight measurement was an electric scale (TANITA HD-390, Tokyo, Japan) and the results were accurate to $0.1 \mathrm{~kg}$. A soft tape was used to measure WC and the participants were measured in fasting states, at the midpoint between the bottom of the rib cage and the uppermost border of the iliac crest, following exhalation. An electronic sphygmomanometer (Omron HBP 1300, Kyoto, Japan) was used for the measurement of blood pressure. Each participant was measured three times for blood pressure, in $5 \mathrm{~min}$ intervals, and the average value was recorded.

Laboratory sample collection and tests included the collection of participants' fingertip blood for testing hemoglobin by a Hemocue 201+ Hemoglobin Analyzer (HemoCue, Angelholm, Sweden). In addition, $6 \mathrm{~mL}$ of fasting venous blood was drawn from participants in the morning and used for testing biochemical and nutritional indicators. Blood glucose, lipids, uric acid, serum vitamin $\mathrm{A}$, vitamin $\mathrm{D}$, and zinc, etc., were detected according to the standard methods provided by the China CDC project group.

\subsection{Diagnostic Criteria and Definitions}

The Chinese "screening standard for malnutrition of school-age children and adolescents" was applied to screen wasting among children by age-specific values [26].

The Chinese "screening for overweight and obesity among school-age children and adolescents" was used to identify overweight and obesity by age-specific values [27]. 
MetS was diagnosed according to the modified criteria of the National Cholesterol Education Program-Adult Treatment Panel III (NCEP-ATP III) for children and adolescents aged 7-17 years [28]. When participants had more than three of the following five components, they were diagnosed as MetS:

(1) Abdominal obesity, where $\mathrm{WC} \geq$ age- and gender-specific 90th percentile [29];

(2) Elevated triglyceride (TG), where TG $\geq 1.24 \mathrm{mmol} / \mathrm{L}$;

(3) Low high-density lipoprotein (HDL), where HDL $\leq 1.03 \mathrm{mmol} / \mathrm{L}$;

(4) Elevated blood pressure, where systolic blood pressure (SBP) or diastolic blood pressure $(\mathrm{DBP}) \geq 90$ th percentile for gender, age, and height [30];

(5) Elevated fasting blood glucose (FBG), where glucose $\geq 6.1 \mathrm{mmol} / \mathrm{L}$.

The following diagnostic criteria and definitions were applied in this study:

Elevated total cholesterol (TC): TC $\geq 5.18 \mathrm{mmol} / \mathrm{L}$ [31]; elevated low high-density lipoprotein (LDL): LDL $\geq 3.36 \mathrm{mmol} / \mathrm{L}$ [31];

Hyperuricemia: serum uric acid (SUA) $\geq 357 \mu \mathrm{mol} / \mathrm{L}$ [21];

Vitamin A deficiency (VAD), marginal deficiency in vitamin A (VAMD), and vitamin A sufficiency were referred to serum retinol levels less than $0.2 \mu \mathrm{g} / \mathrm{mL}, 0.2-0.3 \mu \mathrm{g} / \mathrm{mL}$, and more than $0.3 \mu \mathrm{g} / \mathrm{mL}$, respectively [32];

Vitamin D deficiency (VDD), inadequacy of vitamin D (VDI), and vitamin D sufficiency were defined by serum 25(OH)D levels less than $12 \mathrm{ng} / \mathrm{mL}, 12-20 \mathrm{ng} / \mathrm{mL}$, and more than $20 \mathrm{ng} / \mathrm{mL}$, respectively [33];

Anemia: for participants aged 5-11 years, hemoglobin $<115 \mathrm{~g} / \mathrm{L}$; for participants aged 12-14 years, hemoglobin < $120 \mathrm{~g} / \mathrm{L}$; for male participants aged 15-17 years, hemoglobin < $130 \mathrm{~g} / \mathrm{L}$; for female participants aged 15-17 years, hemoglobin < $120 \mathrm{~g} / \mathrm{L}$ [34];

Zinc deficiency in children and adolescents was defined as serum zinc concentrations $<76.5 \mu \mathrm{g} / \mathrm{dL}[35]$.

The number of overnutrition-related disorders included a count of five MetS components (abdominal obesity, high TG, low HDL, elevated blood pressure, and elevated FBG) and hyperuricemia, high LDL, high TC. The number of undernutrition-related disorders included a count of prevalent vitamin A insufficiency, vitamin D deficiency, anemia, and zinc deficiency.

\subsection{Statistical Analysis}

All statistical analysis was conducted on R software (Version 4.1.0, developed by $\mathrm{R}$ Development Core Team, originated from the Statistics Department of the University of Auckland, Auckland, New Zealand). According to the normality of the continuous variables, they were displayed as either median (interquartile range, IQR) or average (standard deviation, SD). Pearson's chi-square test was used to compare the distribution of different characteristics and metabolic risk factors between gender groups. Multivariate logistic regression analysis was applied to find the related factors for multiple nutritional problems in the research populations.

In order to understand the relevant factors for children's and adolescents' nutritional status, we conducted the multivariate logistic regression analysis by including the following potential factors in the analysis: participants' demographic characteristics, behavioral characteristics, parents' characteristics, and family characteristics. Multivariate Poisson regression analysis was carried out for the overnutrition- and undernutrition-related disorders to test the participants' personal, parental, and household factors. A two-sided $p<0.05$ was utilized to define statistical significance.

\section{Results}

\subsection{Characteristics of Research Populations}

Of the 3025 school-age children and adolescents (7-17 years old) in the study, there were $50.2 \%$ males and $49.8 \%$ females; $34.6 \%$ of the participants were from urban areas and $65.4 \%$ were from rural areas. As displayed in Table 1, the distribution of most characteristics, such as age, age group, living area, physical activity level, screen time, age of mother, age 
of father, education of mother, and education of father, were not significantly different between male and female participants (all $p>0.05$ ). There was a remarkable difference in household size between genders $(p=0.009)$. When it came to anthropometric indexes, male participants had higher height, weight, and SBP than female participants (all $p<0.05$ ), except for DBP, which was not different between males and females (Table 2). There were noticeable differences in many biochemical indices. Male participants had higher levels of FBG, serum uric acid, vitamin $\mathrm{D}$, and serum zinc than did females (all $p<0.05$ ). The TG, $\mathrm{TC}, \mathrm{LDL}$, and HDL of female participants were at higher levels than they were males (all $p<0.05)$. However, there was no significant difference in vitamin A level between genders $(p=0.963)$, as shown in Table 2 .

Table 1. Basic characteristics of the research population.

\begin{tabular}{|c|c|c|c|c|c|}
\hline Variables & $\begin{array}{c}\text { Males (\%) } \\
n=1520\end{array}$ & $\begin{array}{c}\text { Females }(\%) \\
\quad n=1505\end{array}$ & $\begin{array}{c}\text { All } \\
n=3025\end{array}$ & $\begin{array}{l}\text { t or } \chi^{2} \\
\text { Values }\end{array}$ & $p$ Value \\
\hline Age (years) & $11.4 \pm 3.0$ & $11.4 \pm 3.0$ & $11.4 \pm 3.0$ & 0.074 & 0.941 \\
\hline Age group & & & & 0.089 & 0.765 \\
\hline $7-12$ years & $990(65.1)$ & $988(65.6)$ & $1978(65.4)$ & & \\
\hline 13-17 years & $530(34.9)$ & $517(34.4)$ & $1047(34.6)$ & & \\
\hline Living area & & & & 0.001 & 0.982 \\
\hline Urban & $1272(83.7)$ & $1259(83.7)$ & $2531(83.7)$ & & \\
\hline Rural & $248(16.3)$ & $246(16.3)$ & $494(16.3)$ & & \\
\hline Physical activity & & & & 0.613 & 0.434 \\
\hline Low & 907 (59.7) & $919(61.1)$ & $1826(60.4)$ & & \\
\hline High & $613(40.3)$ & $586(38.9)$ & 1199 (39.6) & & \\
\hline Screen time & & & & 3.432 & 0.064 \\
\hline Low & $1320(86.8)$ & $1340(89.0)$ & $2660(87.9)$ & & \\
\hline High & $200(13.2)$ & $165(11.0)$ & $365(12.1)$ & & \\
\hline Age of mother & & & & 0.170 & 0.680 \\
\hline$\leq 37$ (median) & $783(51.5)$ & $764(50.8)$ & $1547(51.1)$ & & \\
\hline$\geq 38$ & $737(48.5)$ & $741(49.2)$ & $1478(48.9)$ & & \\
\hline Age of father & & & & 0.389 & 0.533 \\
\hline$\leq 39$ (median) & $806(53.0)$ & $781(51.9)$ & $1587(52.5)$ & & \\
\hline$\geq 40$ & $714(47.0)$ & $724(48.1)$ & $1438(47.5)$ & & \\
\hline Education of mother & & & & 1.900 & 0.387 \\
\hline Primary school and below & $226(14.9)$ & $206(13.7)$ & $432(14.3)$ & & \\
\hline Middle school & $1007(66.3)$ & $989(65.7)$ & $1996(66.0)$ & & \\
\hline University and above & $287(18.9)$ & $310(20.6)$ & $597(19.7)$ & & \\
\hline Education of father & & & & 0.908 & 0.635 \\
\hline Primary school and below & $134(8.8)$ & $119(7.9)$ & $253(8.4)$ & & \\
\hline Middle school & $1027(67.6)$ & $1034(68.7)$ & $2061(68.1)$ & & \\
\hline University and above & $359(23.6)$ & $352(23.4)$ & $711(23.5)$ & & \\
\hline Household size & & & & 6.907 & 0.009 \\
\hline$\leq 4$ members & $924(60.8)$ & $844(56.1)$ & $1768(58.4)$ & & \\
\hline$\geq 5$ members & $596(39.2)$ & $661(43.9)$ & $1257(41.6)$ & & \\
\hline
\end{tabular}

As noted above, screen time refers to the time of using an electronic screen every day. A low level of screen time is defined as $<2 \mathrm{~h}$ and a high level of screen time is defined as $\geq 2 \mathrm{~h}$. The assessment of physical activities was based on reaching a level of coarsened breathing or a heart rate rise for more than $30 \mathrm{~min}$. An exercise level of $0-3$ days per week was defined as a low physical activity level; an exercise level of more than 4 days per week was defined as a high physical activity level. 
Table 2. Nutritional and biochemistry indicators for the research population.

\begin{tabular}{|c|c|c|c|c|c|}
\hline Variables & $\begin{array}{c}\text { Males } \\
\text { Median (IQR) }\end{array}$ & $\begin{array}{c}\text { Females } \\
\text { Median (IQR) }\end{array}$ & $\begin{array}{c}\text { All } \\
\text { Median (IQR) }\end{array}$ & Z Values & $p$ Value \\
\hline \multicolumn{6}{|l|}{ Anthropometric measurements } \\
\hline Height $(\mathrm{cm})$ & $152.1(138.4,168.0)$ & $152.0(138.0,160.3)$ & $152.1(138.1,162.6)$ & -5.826 & $<0.001$ \\
\hline Weight (kg) & $45.0(32.6,59.4)$ & $42.2(30.9,52.1)$ & $43.5(31.7,55.3)$ & -6.791 & $<0.001$ \\
\hline $\mathrm{WC}(\mathrm{cm})$ & $66.4(59.1,75.1)$ & $62.5(56.1,68.5)$ & $64.0(57.7,71.5)$ & -11.076 & $<0.001$ \\
\hline $\mathrm{SBP}(\mathrm{mmHg})$ & $116.8(108.3,125.5)$ & $113.0(105.3,120.5)$ & $115.0(106.7,122.7)$ & -9.233 & $<0.001$ \\
\hline $\mathrm{DBP}(\mathrm{mmHg})$ & $67.3(62.0,73.3)$ & $67.7(62.7,73.3)$ & $67.7(62.3,73.3)$ & -1.155 & 0.248 \\
\hline \multicolumn{6}{|l|}{ Biochemical indexes } \\
\hline FBG $(\mathrm{mmol} / \mathrm{L})$ & $5.29(5.00,5.59)$ & $5.16(4.90,5.45)$ & $5.22(4.94,5.52)$ & -7.172 & $<0.001$ \\
\hline $\mathrm{TG}(\mathrm{mmol} / \mathrm{L})$ & $0.77(0.60,1.06)$ & $0.83(0.65,1.09)$ & $0.80(0.62,1.06)$ & -5.747 & $<0.001$ \\
\hline $\mathrm{TC}(\mathrm{mmol} / \mathrm{L})$ & $3.95(3.50,4.49)$ & $4.03(3.63,4.54)$ & $4.00(3.59,4.52)$ & -3.282 & 0.001 \\
\hline $\mathrm{LDL}(\mathrm{mmol} / \mathrm{L})$ & $2.16(1.80,2.55)$ & $2.21(1.88,2.59)$ & $2.19(1.84,2.57)$ & -2.448 & 0.014 \\
\hline HDL $(\mathrm{mmol} / \mathrm{L})$ & $1.56(1.30,1.84)$ & $1.60(1.37,1.87)$ & $1.59(1.34,1.86)$ & -2.801 & 0.005 \\
\hline Serum uric acid $(\mu \mathrm{mol} / \mathrm{L})$ & $336.0(276.0,405.0)$ & $297.0(254.0,339.0)$ & $310.0(260.0,369.0)$ & -13.106 & $<0.001$ \\
\hline Vitamin $A(\mu \mathrm{g} / \mathrm{mL})$ & $0.38(0.32,0.43)$ & $0.38(0.32,0.43)$ & $0.38(0.32,0.43)$ & -0.046 & 0.963 \\
\hline Vitamin D (ng/mL) & $16.1(12.8,20.1)$ & $14.5(11.4,18.1)$ & $15.3(12.0,19.1)$ & -8.368 & $<0.001$ \\
\hline Zinc $(\mu \mathrm{g} / \mathrm{dL})$ & $94.0(87.0,102.0)$ & $93.0(85.0,99.0)$ & $93.0(86.0,101.0)$ & -4.386 & $<0.001$ \\
\hline
\end{tabular}

$\mathrm{IQR}=$ interquartile range; $\mathrm{WC}=$ waist circumference; $\mathrm{SBP}=$ systolic blood pressure; $\mathrm{DBP}=$ : diastolic blood pressure; FBG = fasting blood glucose; TG = triglyceride; TC = total cholesterol; LDL = low-density lipoprotein; $\mathrm{HDL}=$ high-density lipoprotein.

\subsection{Nutritional and Health Statuses of School-Age Children and Adolescents}

The nutritional and health statuses of school-age children and adolescents are displayed in Figure 1 and Table 3. Figure 1 is presented according to the sequences of the prevalence of the relevant nutritional and health problems. The prevalence of wasting, overweight, and obesity were $5.5 \%, 14.8 \%$, and $12.7 \%$, respectively. MetS was prevalent among $5.1 \%$ of the participants. The prevalence of the five MetS components, including abdominal obesity, high TG, low HDL, elevated blood pressure, and elevated FBG, were $18.7 \%, 15.0 \%, 4.1 \%, 41.3 \%$, and $3.5 \%$, respectively. Among the study participants, $29.5 \%$ had hyperuricemia. The prevalence of high LDL among all participants was $4.8 \%$. The prevalence of high TC among all participants was $7.4 \% ; 0.9 \%$ of the participants were VAD, $14.6 \%$ had marginal vitamin D deficiency, and $84.5 \%$ were sufficient in vitamin $\mathrm{A}$ levels; $25.1 \%$ of the participants had VDD, $54.5 \%$ had inadequate VDD, and $20.4 \%$ were sufficient in vitamin D levels. The prevalence of anemia among all participants was $4.0 \%$. The prevalence of zinc deficiency was $4.8 \%$. There were significant differences between genders in the prevalence of weight groups, MetS, hyperuricemia, high TC, and vitamin D conditions, as shown in Table 3.

After age group stratification, we compared the nutritional status within each age group and between the age groups, as shown in the Supplementary Materials, Table S1. The proportions for weight groups, vitamin $\mathrm{A}$, and vitamin $\mathrm{D}$ were significantly different between participants in the 7-12 years age group and the 13-17 years age group $(p<0.001)$. The 13-17 years age group had a significant higher possibility of hyperuricemia and anemia than did the $7-12$ years age group (both $p<0.05$ ).

\subsection{Related Factors Associated with Various Nutritional Diseases}

In Table 4, the multivariate logistic regression of multiple nutritional diseases (MetS, hyperuricemia, vitamin A insufficiency, vitamin D deficiency, anemia, and zinc deficiency) are displayed. Table 4 includes the most common factors of demographic characteristics, behavioral characteristics, parents' characteristics, and family characteristics. These common factors were considered in the multivariate logistic analysis. The adjusted odds ratios (AORs), along with their $95 \%$ confidence intervals ( $95 \% \mathrm{CI}$ ) and $p$ values, are portrayed in Table 4. 


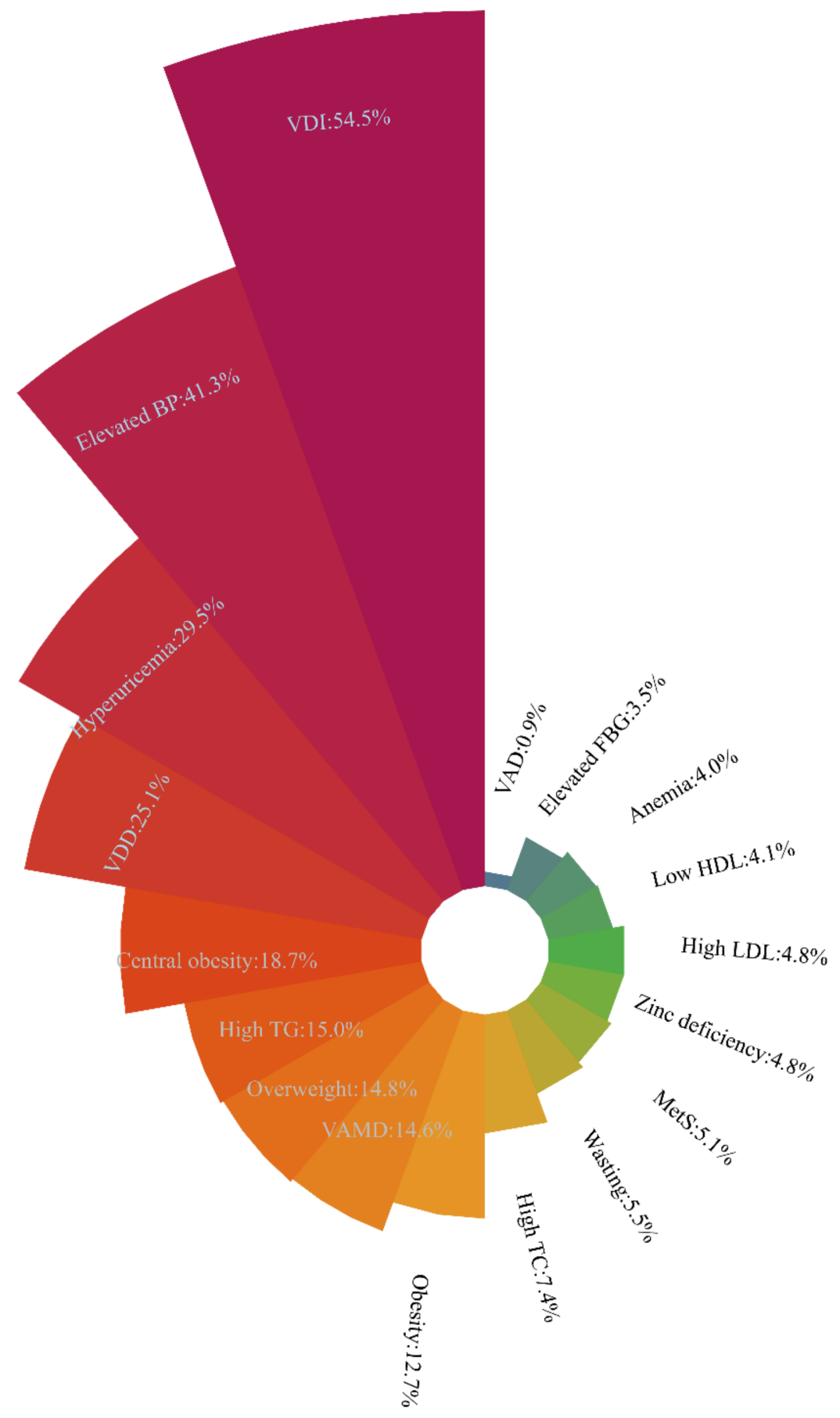

Figure 1. Sequence of prevalence of the relevant nutritional and health problems among school-age children and adolescents. VDI = vitamin D inadequacy; elevated BP = elevated blood pressure; $\mathrm{VDD}=$ vitamin D deficiency; high TG = high triglyceride; VAMD = vitamin A marginal deficiency; high TC = high total cholesterol; MetS = metabolic syndrome; high LDL = high low-density lipoprotein; low HDL = low high-density lipoprotein; elevated FBG = elevated fasting blood glucose; $\mathrm{VAD}=$ vitamin A deficiency. MetS and its five components (central obesity, high TG, low HDL, elevated BP, and elevated FBG) were diagnosed according to the modified criteria of the National Cholesterol Education Program-Adult Treatment Panel III (NCEP-ATP III) for children and adolescents aged $7-17$ years. 
Table 3. Prevalence of various categories of nutritional status among school-age children and adolescents in Jiangsu.

\begin{tabular}{|c|c|c|c|c|c|}
\hline Nutritional Status & Males $n(\%)$ & Female $n(\%)$ & Total $n(\%)$ & $x^{2}$ Values & $p$ Value \\
\hline Weight groups & & & & 102.298 & $<0.001$ \\
\hline Wasting & $96(6.3)$ & $70(4.7)$ & $166(5.5)$ & & \\
\hline Normal weight & $893(58.8)$ & $1136(45.5)$ & $2029(67.1)$ & & \\
\hline Overweight & $271(17.8)$ & $176(11.7)$ & $447(14.8)$ & & \\
\hline Obesity & $260(17.1)$ & $123(8.2)$ & $383(12.7)$ & & \\
\hline MetS & & & & 6.215 & 0.013 \\
\hline No & 1427 (93.9) & 1443 (95.9) & $2870(94.9)$ & & \\
\hline Yes & $93(6.1)$ & $62(4.1)$ & $155(5.1)$ & & \\
\hline Abdominal obesity & & & & 18.075 & $<0.001$ \\
\hline No & $1190(78.3)$ & $1269(84.3)$ & $2459(81.3)$ & & \\
\hline Yes & $330(21.7)$ & $236(15.7)$ & $566(18.7)$ & & \\
\hline High TG & & & & 1.403 & 0.236 \\
\hline No & $1304(85.8)$ & $1268(84.3)$ & $2572(85.0)$ & & \\
\hline Yes & $216(14.2)$ & $237(15.7)$ & $453(15.0)$ & & \\
\hline Low HDL & & & & 3.160 & 0.075 \\
\hline No & $95.3(1448)$ & $1453(96.5)$ & $2901(95.9)$ & & \\
\hline Yes & $72(4.7)$ & $52(3.5)$ & $124(4.1)$ & & \\
\hline Elevated BP & & & & 0.159 & 0.690 \\
\hline No & $887(58.4)$ & $889(59.1)$ & $1179(58.7)$ & & \\
\hline Yes & $633(41.6)$ & $616(40.9)$ & 1249 (41.3) & & \\
\hline Elevated FBG & & & & 15.868 & $<0.001$ \\
\hline No & $1446(95.1)$ & $1472(97.8)$ & $2918(96.5)$ & & \\
\hline Yes & $74(4.9)$ & $33(2.2)$ & $107(3.5)$ & & \\
\hline Hyperuricemia & & & & 194.297 & $<0.001$ \\
\hline No & $897(59.0)$ & $1236(82.1)$ & $2133(70.5)$ & & \\
\hline Yes & $623(41.0)$ & $269(17.9)$ & $892(29.5)$ & & \\
\hline High LDL & & & & 0.432 & 0.511 \\
\hline No & $1451(95.5)$ & $1429(95.0)$ & $2880(95.2)$ & & \\
\hline Yes & $69(4.5)$ & $76(5.0)$ & $145(4.8)$ & & \\
\hline High TC & & & & 7.727 & 0.005 \\
\hline No & 1427 (93.9) & $1373(91.2)$ & $2800(92.6)$ & & \\
\hline Yes & $93(6.1)$ & $132(8.8)$ & $225(7.4)$ & & \\
\hline Vitamin A & & & & 0.049 & 0.976 \\
\hline Sufficiency & $1285(84.5)$ & $1272(84.5)$ & $2557(84.5)$ & & \\
\hline Marginal deficiency & $222(14.6)$ & $219(14.6)$ & $441(14.6)$ & & \\
\hline Deficiency & $13(0.9)$ & $14(0.9)$ & $27(0.9)$ & & \\
\hline Vitamin D & & & & 68.349 & $<0.001$ \\
\hline Sufficiency & $383(25.2)$ & $233(15.5)$ & $616(20.4)$ & & \\
\hline Inadequacy & 835 (54.9) & $815(54.2)$ & $1650(54.5)$ & & \\
\hline Deficiency & $302(19.9)$ & $457(30.4)$ & $759(25.1)$ & & \\
\hline Anemia & & & & 31.863 & $<0.001$ \\
\hline No & $1490(98.0)$ & $1415(94.0)$ & $2905(96.0)$ & & \\
\hline Yes & $30(2.0)$ & $90(6.0)$ & $120(4.0)$ & & \\
\hline Zinc deficiency & & & & 3.417 & 0.065 \\
\hline No & $1458(95.9)$ & $1422(94.5)$ & $2880(95.2)$ & & \\
\hline Yes & $62(4.1)$ & $83(5.5)$ & $145(4.8)$ & & \\
\hline
\end{tabular}

MetS = metabolic syndrome; high TG = high triglyceride; low HDL = low high-density lipoprotein; elevated $\mathrm{BP}=$ elevated blood pressure; elevated FBG = elevated fasting blood glucose; high LDL = high low-density lipoprotein; high TC = high total cholesterol.

We found that age groups and weight groups were significantly associated with MetS. Compared to the 7-12 years age group, the 13-17 years age group was more prone to have MetS (AOR $=1.79,95 \%$ CI: $1.17-2.73, p=0.007)$. Participants in the overweight groups were more likely to have MetS (OR $=7.47,95 \%$ CI: 4.26-13.11, $p<0.001$ ), compared to those in the reference group (in terms of wasting and normal weight). Furthermore, participants in the obesity group were more likely to have MetS than were those in the reference group $(\mathrm{OR}=41.14,95 \%$ CI: 25.05-67.56, $p<0.001)$. 
Table 4. Multivariate logistic regression analysis of various nutritional disorders among school-age children and adolescents in Jiangsu Province.

\begin{tabular}{|c|c|c|c|c|c|c|c|c|c|c|c|c|}
\hline \multirow[b]{2}{*}{ Characteristics } & \multicolumn{2}{|c|}{ Metabolic Syndrome } & \multicolumn{2}{|c|}{ Hyperuricemia } & \multicolumn{2}{|c|}{ Vitamin A Insufficiency } & \multicolumn{2}{|c|}{ Vitamin D Deficiency } & \multicolumn{2}{|c|}{ Anemia } & \multicolumn{2}{|c|}{ Zinc Deficiency } \\
\hline & AOR $(95 \% \mathrm{CI})$ & $p$ Value & AOR $(95 \% \mathrm{CI})$ & $p$ Value & AOR $(95 \% \mathrm{CI})$ & $p$ Value & AOR $(95 \% \mathrm{CI})$ & $p$ Value & AOR $(95 \% \mathrm{CI})$ & $p$ Value & AOR $(95 \% \mathrm{CI})$ & $p$ Value \\
\hline \multicolumn{13}{|l|}{ Sex } \\
\hline Males (reference) & 1.00 & - & 1.00 & - & 1.00 & 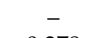 & 1.00 & - & 1.00 & - & 1.00 & - \\
\hline \multirow{2}{*}{\multicolumn{13}{|c|}{ Age group }} \\
\hline & & & & & & & & & & & & \\
\hline 13-17 years & $1.79(1.17-2.73)$ & 0.007 & $5.09(4.13-6.27)$ & $<0.001$ & $0.34(0.26-0.45)$ & $<0 . \overline{0} 01$ & $2.53(2.09-3.08)$ & $<0.001$ & $1.82(1.20-2.77)$ & $0 . \overline{0}$ & $\begin{array}{c}1.00 \\
0.94(0.63-1.41)\end{array}$ & $0 . \overline{774}$ \\
\hline \multicolumn{13}{|l|}{ Living area } \\
\hline Urban & 1.00 & - & 1.00 & - & 1.00 & - & 1.00 & - & 1.00 & - & 1.00 & - \\
\hline Rural & $0.76(0.43-1.34)$ & 0.345 & $0.97(0.75-1.25)$ & 0.796 & $1.24(0.95-1.62)$ & 0.116 & $1.14(0.89-1.44)$ & 0.298 & $1.43(0.89-2.31)$ & 0.142 & $1.80(1.21-2.68)$ & 0.004 \\
\hline Weight group & & & & & & & & & & & & \\
\hline Others (reference) & 1.00 & - & 1.00 & - & 1.00 & - & 1.00 & - & 1.00 & - & 1.00 & - \\
\hline Overweight & $\begin{array}{c}7.47 \\
(4.26-13.11)\end{array}$ & $<0.001$ & $1.94(1.53-2.46)$ & $<0.001$ & $0.63(0.46-0.87)$ & $<0.001$ & $1.02(0.80-1.31)$ & 0.877 & $0.71(0.39-1.28)$ & 0.255 & $0.78(0.46-1.32)$ & 0.357 \\
\hline Obesity & $\begin{array}{c}41.14 \\
(25.05-67.56)\end{array}$ & $<0.001$ & $4.38(3.38-5.67)$ & $<0.001$ & $0.37(0.25-0.55)$ & 0.005 & $0.85(0.64-1.13)$ & 0.269 & $0.37(0.15-0.93)$ & 0.034 & $0.81(0.45-1.44)$ & 0.466 \\
\hline \multicolumn{13}{|l|}{ Physical activity } \\
\hline Low & 1.00 & - & 1.00 & - & 1.00 & - & 1.00 & - & 1.00 & - & 1.00 & - \\
\hline High & $1.31(0.91-1.88)$ & 0.146 & $1.19(0.99-1.43)$ & 0.061 & $0.93(0.75-1.16)$ & 0.528 & $0.69(0.58-0.83)$ & $<0.001$ & $1.07(0.73-1.56)$ & 0.746 & $1.25(0.89-1.77)$ & 0.204 \\
\hline \multicolumn{13}{|l|}{ Screen time } \\
\hline Low & 1.00 & - & 1.00 & - & 1.00 & - & 1.00 & - & 1.00 & - & 1.00 & - \\
\hline High & $0.60(0.33-1.10)$ & 0.097 & $0.91(0.69-1.19)$ & 0.487 & $1.45(1.09-1.94)$ & 0.011 & $1.40(1.09-1.80)$ & 0.008 & $1.25(0.73-2.13)$ & 0.420 & $0.78(0.46-1.32)$ & 0.360 \\
\hline \multicolumn{13}{|l|}{ Age of mother } \\
\hline$\leq 36$ & 1.00 & - & 1.00 & - & 1.00 & - & 1.00 & - & 1.00 & - & 1.00 & - \\
\hline \multirow{2}{*}{\multicolumn{13}{|c|}{ Age of father }} \\
\hline & & & & & & & & & & & & \\
\hline$\leq 36$ & 1.00 & - & 1.00 & - & 1.00 & - & 1.00 & - & 1.00 & - & 1.00 & - \\
\hline$\geq 37$ & $0.97(0.58-1.63)$ & 0.901 & $0.94(0.73-1.23)$ & 0.668 & $1.07(0.79-1.45)$ & 0.662 & $1.03(0.80-1.33)$ & 0.788 & $0.75(0.44-1.27)$ & 0.285 & $0.82(0.49-1.36)$ & 0.443 \\
\hline \multicolumn{7}{|l|}{ Education of mother } & & & & & & \\
\hline $\begin{array}{l}\text { Primary school and below } \\
\text { Middle school }\end{array}$ & $1.08(0.58-2.03)$ & $0 . \overline{804}$ & $0.94(0.71-1.25)$ & $0 . \overline{693}$ & $\begin{array}{c}1.00 \\
0.64(0.47-0.88)\end{array}$ & $0 \overline{005}$ & $\begin{array}{c}1.00 \\
0.68(0.52-0.89)\end{array}$ & 0.004 & $\begin{array}{c}1.00 \\
1.27(0.70-29)\end{array}$ & - & $\begin{array}{l}1.00 \\
0.56\end{array}$ & - \\
\hline $\begin{array}{l}\text { Middle school } \\
\text { University and above }\end{array}$ & $1.09(0.47-2.50)$ & $\begin{array}{l}0.804 \\
0.847\end{array}$ & $1.23(0.82-1.83)$ & 0.693 & $\begin{array}{l}0.64(0.47-0.88) \\
0.55(0.35-0.89)\end{array}$ & 0.005 & $0.68(0.52-0.89)$ & 0.004 & $1.27(0.70-2.29)$ & 0.438 & $0.95(0.58-1.56)$ & 0.847 \\
\hline \multicolumn{13}{|l|}{ Education of father } \\
\hline Primary school and below & 1.00 & - & 1.00 & - & 1.00 & - & 1.00 & - & 1.00 & - & 1.00 & - \\
\hline Middle school & $0.92(0.45-1.87)$ & 0.817 & $0.86(0.61-1.22)$ & 0.397 & $1.10(0.74-1.64)$ & 0.626 & $1.17(0.84-1.62)$ & 0.364 & $1.06(0.51-2.20)$ & 0.886 & $0.72(0.41-1.28)$ & 0.268 \\
\hline University and above & $0.58(0.24-1.40)$ & 0.228 & $0.63(0.41-0.97)$ & 0.035 & $0.73(0.43-1.22)$ & 0.223 & $0.97(0.63-1.48)$ & 0.883 & $1.03(0.51-2.20)$ & 0.942 & $0.47(0.20-1.09)$ & 0.078 \\
\hline \multicolumn{13}{|l|}{ Household size } \\
\hline$\leq 4$ members & 1.00 & - & 1.00 & - & 1.00 & - & 1.00 & - & 1.00 & - & 1.00 & - \\
\hline$\geq 5$ members & $1.20(0.83-1.73)$ & 0.327 & $0.93(0.77-1.12)$ & 0.465 & $1.20(0.97-1.47)$ & 0.088 & $1.25(1.05-1.49)$ & 0.014 & $1.10(0.75-1.62)$ & 0.609 & $1.30(0.92-1.83)$ & 0.136 \\
\hline
\end{tabular}


With respect to hyperuricemia, a participant's sex, age group, weight group, age of the mother, and education of the father were relevant factors. Females had significantly less likelihood of hyperuricemia than did males (OR $=0.30,95 \%$ CI: $0.25-0.36, p<0.001$ ). Participants in the 13-17 years age group had a higher possibility of hyperuricemia than did those in the 7-12 years age group (OR $=5.09,95 \% \mathrm{CI}: 4.13-6.27, p<0.001)$. The overweight and obesity groups were both higher in the prevalence of hyperuricemia than were other groups $(\mathrm{OR}=1.94,95 \% \mathrm{CI}: 1.53-2.46$, and $\mathrm{OR}=4.38,95 \% \mathrm{CI}: 3.38-5.67$, respectively). Compared to the participants whose mothers were younger than 37 years, the participants whose mothers were older than 37 years were more likely to have hyperuricemia $(\mathrm{OR}=1.46$, 95\% CI: 1.13-1.89, $p=0.004)$. Participants whose father's education was university and above were less likely to have hyperuricemia than were those whose father's education was primary school and below $(\mathrm{OR}=0.63,95 \% \mathrm{CI}: 0.41-0.97, p=0.035)$.

When focusing on vitamin A insufficiency, a participant's age group, weight group, screen time, and education of the mother were the relevant factors. Participants in the 13-17 years age group were less likely to have a prevalence of vitamin A insufficiency, when compared with the those in the $7-12$ years age group (OR $=0.34,95 \% \mathrm{CI}: 0.26-0.45$, $p<0.001)$. Participants who were overweight had less risk of vitamin A insufficiency than did those in the reference group (in terms of wasting and normal weight, OR $=0.63,95 \%$ CI: 0.46-0.87). Obesity subjects also had less risk of vitamin A insufficiency than did those in the reference group ( $\mathrm{OR}=0.37,95 \% \mathrm{CI}: 0.25-0.55, p=0.005)$. In addition, participants with higher screen time were prone to have vitamin A insufficiency $(\mathrm{OR}=1.45,95 \% \mathrm{CI}$ : $1.09-1.94, p=0.011)$. Children and adolescents whose mothers had higher education levels were less likely to have vitamin A insufficiency, compared to those whose mothers had primary school education or below; the OR were 0.64 and 0.55 , respectively.

With reference to VDD, a participant's sex, age group, physical activity, screen time, education of the mother, and household size were the relevant factors. Females were more likely to have VDD than males, with AOR $=1.80,95 \% \mathrm{CI}: 1.51-2.15, p<0.001$. The 13-17 years participants had a higher risk of being VDD than did the 7-12 years participants $(\mathrm{OR}=2.53,95 \% \mathrm{CI}: 2.09-3.08, p<0.001)$. Remarkably, participants with a high level of physical activity were less likely to have VDD (AOR = 0.69, 95\% CI: 0.58-0.83, $p<0.001)$. Subjects with high screen time were associated with an increased risk of VDD (AOR $=1.40,95 \%$ CI: 1.09-1.80, $p=0.008)$. In addition, VDD was related to participants' mothers' education levels (middle school vs. primary school and below: AOR =0.68, 95\% CI: 0.52-0.89; university and above vs. primary school and below: $A O R=0.58,95 \% \mathrm{CI}$ : $0.40-0.86)$. Those participants whose household size was $\geq 5$ members were more prone to have VDD than were those whose household size was $\leq 4$ members (AOR $=1.25,95 \%$ $\mathrm{CI}=1.05-1.49, p=0.014)$.

When considering anemia, the sex, age group, and obesity of participants were the relevant factors. Sex was associated with anemia (females were more likely to have anemia, with $\mathrm{AOR}=2.95,95 \% \mathrm{CI}=1.95-4.51, p<0.001)$, as well the age group (13-17 years: $\mathrm{AOR}=1.82,95 \% \mathrm{CI}: 1.20-2.77, p=0.005)$. Inversely, obesity groups were less likely to show prevalence in anemia $(\mathrm{AOR}=0.37,95 \% \mathrm{CI}: 0.15-0.93, p=0.034)$. For zinc deficiency, participants living in the rural area had a higher risk than did those who lived in urban areas $(\mathrm{AOR}=1.80,95 \% \mathrm{CI}: 1.21-2.68, p=0.004)$.

\subsection{The Relevant Factors of Overnutrition- and Undernutrtion-Related Disorders}

The Poisson regression analysis took the amount of overnutrition-related disorders (i.e., the prevalent five MetS components and hyperuricemia, high LDL, and high TC) and undernutrition-related disorders (i.e., vitamin A insufficiency, VDD, anemia, and zinc deficiency) as dependent variables to test the personal, parental, and household factors. We found that the factors of sex, age group, and weight group were related to overnutritionrelated disorders, seen in Table 5 . Females were less likely inclined to overnutrition-related disorders $(\mathrm{AOR}=0.81,95 \% \mathrm{CI}: 0.51-0.85, p<0.05)$. The older age group (13-17 years) and the overweight and obesity participants were more prone to have a variety of overnutrition- 
related disorders (AOR $=1.44,1.66$ and 2.77, respectively, all $p<0.05)$. With respect to undernutrition-related disorders, we found that a participant's sex, age, living area, weight group, physical activity, screen time, education of mother, and household size were the relevant factors. Females, older age group (13-17 years), rural areas, higher screen time, and large household size ( $\geq 5$ members) were significantly and positively associated with the occurrence of undernutrition-related disorders (all AOR > 1). Conversely, participants who were overweight or obese, or who had a high level of physical activity or a mother with higher education, were less prone to clustering in undernutrition-related disorders. (All AOR $<1$ and all $p<0.05$ ).

Table 5. Multivariate Poisson regression analysis of overnutrition- and undernutrition-related disorders among school-age children and adolescents in Jiangsu Province.

\begin{tabular}{|c|c|c|c|c|}
\hline \multirow[b]{2}{*}{ Characteristics } & \multicolumn{2}{|c|}{$\begin{array}{c}\text { Overnutrition-Related } \\
\text { Disorders }\end{array}$} & \multicolumn{2}{|c|}{$\begin{array}{c}\text { Undernutrition-Related } \\
\text { Disorders }\end{array}$} \\
\hline & IRR $(95 \% C I)$ & $p$ Value & IRR $(95 \% C I)$ & $p$ Value \\
\hline \multicolumn{5}{|l|}{ Sex } \\
\hline Males (reference) & 1.00 & - & 1.00 & - \\
\hline Females & $0.81(0.51-0.85)$ & $<0.001$ & $1.33(1.20-1.48)$ & $<0.001$ \\
\hline \multicolumn{5}{|l|}{ Age group } \\
\hline 7-12 years (reference) & 1.00 & - & 1.00 & - \\
\hline $13-17$ years & $1.44(1.35-1.54)$ & $<0.001$ & $1.17(1.04-1.32)$ & 0.008 \\
\hline \multicolumn{5}{|l|}{ Living area } \\
\hline Urban & 1.00 & - & 1.00 & - \\
\hline Rural & $1.00(0.91-1.08)$ & 0.939 & $1.21(1.06-1.38)$ & 0.005 \\
\hline \multicolumn{5}{|l|}{ Weight group } \\
\hline Others (reference) & 1.00 & - & 1.00 & - \\
\hline Overweight & $1.66(1.53-1.79)$ & $<0.001$ & $0.86(0.73-1.00)$ & 0.05 \\
\hline Obesity & $2.77(2.58-2.97)$ & $<0.001$ & $0.67(0.55-0.81)$ & $<0.001$ \\
\hline \multicolumn{5}{|l|}{ Physical activity } \\
\hline Low & 1.00 & - & 1.00 & - \\
\hline High & $1.05(0.99-1.12)$ & 0.095 & $0.89(0.80-0.99)$ & 0.027 \\
\hline \multicolumn{5}{|l|}{ Screen time } \\
\hline Low & 1.00 & - & 1.00 & - \\
\hline High & $0.94(0.86-1.03)$ & 0.202 & $1.23(1.06-1.42)$ & 0.005 \\
\hline \multicolumn{5}{|l|}{ Age of mother } \\
\hline$\leq 37$ & 1.00 & - & 1.00 & - \\
\hline$\geq 38$ & $1.07(0.98-1.16)$ & 0.122 & $0.99(0.86-1.15)$ & 0.946 \\
\hline \multicolumn{5}{|l|}{ Age of father } \\
\hline$\leq 36$ & 1.00 & - & 1.00 & - \\
\hline$\geq 37$ & $0.98(0.90-1.07)$ & 0.658 & $0.99(0.85-1.15)$ & 0.852 \\
\hline \multicolumn{5}{|l|}{ Education of mother } \\
\hline Primary school and below & 1.00 & - & 1.00 & - \\
\hline Middle school & $0.99(0.90-1.08)$ & 0.759 & $0.81(0.70-0.94)$ & 0.006 \\
\hline University and above & $1.03(0.91-1.18)$ & 0.631 & $0.68(0.54-0.89)$ & 0.001 \\
\hline \multicolumn{5}{|l|}{ Education of father } \\
\hline Primary school and below & 1.00 & - & 1.00 & - \\
\hline Middle school & $1.01(0.90-1.13)$ & 0.913 & $1.04(0.86-1.26)$ & 0.689 \\
\hline University and above & $0.90(0.77-1.04)$ & 0.135 & $0.83(0.65-1.07)$ & 0.147 \\
\hline \multicolumn{5}{|l|}{ Household size } \\
\hline$\leq 4$ members & 1.00 & - & 1.00 & - \\
\hline$\geq 5$ members & $1.02(0.96-1.09)$ & 0.435 & $1.17(1.05-1.29)$ & 0.004 \\
\hline
\end{tabular}

Overnutrition-related disorders: the prevalent five MetS components and hyperuricemia, high LDL, and high TC. Undernutrition-related disorders: prevalent vitamin A insufficiency, vitamin D deficiency, anemia, and zinc deficiency. IRR: incidence rate rations. 95\%CI: 95\% confidence interval.

\section{Discussion}

The present study illustrated the multiple nutritional statuses of school-age children and adolescents in Jiangsu Province, China. The presence of the double burden 
of malnutrition was common among the children and adolescents. To be more specific, overnutrition-related diseases such as overweight, obesity, MetS, hyperuricemia, high LDL and high TC, and undernutrition-related diseases (vitamin A insufficiency, VDD, anemia, and zinc deficiency) were prevalent in the 7-17 years age group of participants. Demographic characteristics, behavior characteristics, parents' characteristics, and family characteristics were associated with different malnutrition disorders.

In this study, 5.5\% of school-age children and adolescents in Jiangsu Province showed prevalence in wasting, according to the Chinese standard, and males were more prone to wasting than were females (6.3\% vs. 4.7\%). Based on data from 2014 Chinese National Surveys on Students Constitution and Health, wasting was present in $5.5 \%$ of Chinese children and adolescents, which was consistent with the findings in our study [36]. In present study, the prevalence of overweight and obesity was $14.8 \%$ and $12.7 \%$, respectively. The prevalence of both overweight and obesity was higher in males than in females. Results from the 2015 China Health and Nutrition Survey (CHNS) showed that, generally, overweight prevalence was $15.43 \%$ and obesity prevalence was $11.06 \%$ among children and adolescents aged 6 to 17 years [37]. Data from NHANES indicated that obesity prevalence among America youth aged 2-19 years, from 2015-2016, was 18.5\% [38].

Overweight and obesity have become a serious nutritional problem in children all around the world $[39,40]$. The prevalence of MetS in this study's participants was 5.1\%, while the results of the CHNS yielded an overall 3.37\% prevalence of MetS [18]. The Jiangsu Province's level of prevalence of MetS in school-age children and adolescents was higher than the national average. This indicated that urgent measures must be taken to contain MetS. Among school-age children and adolescents, 29.5\% had hyperuricemia $(\geq 357 \mu \mathrm{mol} / \mathrm{L})$. The overall prevalence of hyperuricemia among American adults had increased from $18.0 \%$ in 1988 to $21.4 \%$ in 2008 [41], which suggested a high prevalence level of hyperuricemia in a large number of children and adolescents.

In our study, we found VAD and marginal deficiency in vitamin A were prevalent, respectively, in $0.9 \%$ and $14.6 \%$ of the participants. Similarly, the national results of the China Nutrition and Health Surveillance of Children and Lactating Mothers, in 2016-2017, showed that the prevalence of VAD and the marginal deficiency of vitamin A was $0.96 \%$ and $14.71 \%$, respectively [42]. The prevalence of VAD and marginal deficiency in vitamin A among school-age children and adolescents in Jiangsu Province were at the national average level. Based on these findings, vitamin A marginal deficiency was the main form of vitamin A insufficiency in Chinese children. The prevalence of VDD and inadequacy of vitamin D were $25.1 \%$ and $54.5 \%$, respectively. In spite of different diagnostic criteria, VDD was very common among school-age children $[43,44]$. Sufficient sunlight, dietary vitamin $\mathrm{D}$ and vitamin D supplements are recommended for children.

Our study indicated that the presence of anemia in children and adolescents aged 7-17 years was $4.0 \%$. Anemia was more common in girls than in boys, at $6.0 \%$ vs. $2.0 \%$, respectively. These results were consistent with data from the Chinese National Nutrition and Health Survey (CNNHS) [45], where $4.8 \%$ of children had zinc deficiency and the prevalence of zinc deficiency was not different between genders. Zinc is an essential microelement for growth and development in children, but zinc deficiency is still a serious nutritional problem, especially in school-age children [13]. These findings indicated that multiple overnutrition-related diseases and undernutrition-related diseases coexisted in the surveyed areas.

In addition, we analyzed the roles of different factors, such as demographic characteristics, behavioral characteristics, parents' characteristics, and family characteristics in the development of different malnutrition diseases. Age and weight are related to MetS. Participating adolescents over 13 years, obese participants, and overweight participants are more likely to suffer from MetS. Other research has also shown that age and obesity can increase the risk of developing MetS [46]. These results indicate that more attention should be paid to obese and overweight children, and to adolescents aged 13-17 years. 
Based on our study, age, weight and the age of mothers were positively associated with hyperuricemia. Females and children whose mothers had high education levels were less likely to have hyperuricemia. The level of uric acid increased with age in children aged 5-14 years in southeast China [47]. One study showed that boys had higher mean serum uric acid concentrations than girls [19], and this difference was consistent with our results. Age and education of the mother were new influencing factors for hyperuricemia in children. These results support the view that family factors, especially factors pertaining to mothers, may have some influence on the development of hyperuricemia in children.

When focusing on vitamin A insufficiency, age, weight, screen time, and the education of mothers are relevant factors. Older, overweight, and obese children, and those whose mothers have higher education levels, are less likely to be insufficient in vitamin A. Similarly, a meta-analysis reported that a prevalence of VAD and marginal VAD both decreased with increasing age in Chinese children [9]. Furthermore, a Chinese nationally representative study reported that the average of serum retinol concentration was notably higher in over-nutrified than non-over nutrified children and adolescents [48].

The screen time of children was positively associated with vitamin A insufficiency. This can be understood by the fact that vitamin A is the key nutrient implicated in maintaining the function of normal vision; prolonged eye use may consume vitamin A [49,50]. Vitamin D, with its influence on calcium and phosphorus homeostasis, as well as bone health and immunity health, is the essential micronutrient in the growth and development of children [51]. VDD is associated with numerous adverse health issues, such as MetS [52], dyslipidemia [53], and cardiovascular diseases [54]. In our study, sex, age, physical activity, screen time, the education of mothers, and household sizes were related to VDD. The factors of female gender, age more than 13 years, high screen time, and large household size were positively associated with VDD in this population.

One Chinese study investigated VDD among 460,537 children and concluded that the factor of female gender contributed to VDD and vitamin D insufficiency [55]. Girls and boys have different fat distributions and girls have a higher physiological level of estrogens that play certain roles in vitamin D metabolism [56]. Older children have a higher risk of VDD, which can be demonstrated by the facts that muscle mass increases after puberty and muscle is a storage site for vitamin D metabolites [57,58]. Higher screen time means less sun exposure, which can affect vitamin D synthesis [59]. Meanwhile, family factors, such as education of mothers and household sizes, may have some roles in the development of VDD.

In our research, the anemia-related factors were sex, age, and weight. Our results accorded with the CNNHS in 2010-2012, which analyzed the factors associated with anemia in Chinese children and adolescents [45]. That study found that girls and older children were less likely to have anemia and that obese children had a significantly lower prevalence of anemia than did those who had normal or low body weight, which could be due to a better dietary nutritional intake in overweight or obese people [45].

The present study found that children living in rural areas were more likely to be zinc deficient than were those who lived in urban areas. A systematic review of the literature came to the same conclusion [60]. Zinc deficiency may result from low zinc dietary intake and/or the reduction in bioavailability of zinc. Consumption of foods with high phytate content (e.g., rice and wheat) in the rural areas may lead to low zinc absorption and reduced bioavailability [61].

Moreover, the Poisson regression analysis results showed that girls seemed to have a higher risk of undernutrition-related disorders and less risk of overnutrition-related disorders than boys, which was consistent with previous evidence [62]. There is evidence that girls are more vulnerable than boys to disadvantage in nutrient intake, due to China's "son preference" norm [63]. Novel findings in our study revealed that the ages of children, and adolescents aged 13-17 years, were related to multiple overnutrition and undernutrition disorders. In this study, adolescents aged 13-17 years had a higher prevalence of overweight, MetS, and hyperuricemia. On the other side, adolescents aged 13-17 years 
had a higher prevalence of VDD and anemia. Therefore, we should pay special attention to the various nutritional and health problems of adolescents in puberty and late puberty. Children's caregivers in poor areas of China have a lack of feeding information and cannot give their children complementary foods in a proper way, including proper starting times, frequency, and quality [64]. Lower education among rural caregivers was another risk factor in children's stunting. This result was consistent with previous studies [65].

Furthermore, childhood obesity is linked to serious complications in adulthood, which include an increased risk of ill health and premature death [66]. The high prevalence and dramatically rising trend in childhood obesity suggest that aggressive approaches in prevention and treatment should be pursued to reduce the attendant health and social consequences of obesity. One previous study indicated that children and adolescents aged 6-17 who participate in moderate to vigorous physical activities are likely to experience multiple positive health outcomes [67]. Thus, providing sufficient physical activity time is essential for children's and adolescents' health. Screen time, including the daily use of mobile phones, TV watching, and playing computer and video games, was related to the nutritional status of children [68], and especially to vitamin A insufficiency and VDD. For children's nutrition and vision health, it is necessary to strictly limit everyday screen time. In addition, domestic factors have influences on children's nutritional health. For example, the significant positive effects of a family economy on the weight of children and adolescents have been gradually and persistently increasing [69].

It is noted that this study does have some limitations. First, this study did not take into consideration dietary factors and some other influences, such as environmental factors or genetic susceptibility; however, these factors may raise issues affecting different nutritional and health problems among children and adolescents. In future studies, we will further explore the roles of these factors in the nutritional status of children. Second, this study, as a cross-sectional design, only provides clues on the relationships between nutritional problems and relevant factors; thus, further studies are needed to verify these relationships. However, through rigorous surveys and scientific sampling methods, a large regional representative sample of participants among eastern Chinese children and adolescents can present findings on multiple nutritional issues.

\section{Conclusions}

The presence of the double burdens of malnutrition are common among school-age children and adolescents in Jiangsu Province in eastern China. Demographic characteristics, behavior characteristics, parents' characteristics, and family characteristics were associated with multiple malnutrition disorders. Health education, focusing on behavioral intervention and nutritional education of children, and on their living environments, may be a practical strategy in preventing multiple malnutrition disorders in school-age children and adolescents.

Supplementary Materials: The following supporting information can be downloaded at https: / / www.mdpi.com/article/10.3390/nu14040758/s1: Table S1: Prevalence of various nutritional status in Jiangsu Province children and adolescents aged 7-12 years and 13-17 years.

Author Contributions: Y.Z. and Y.D. participated in the conceptualization and methodology of the study; T.T. and Y.W. carried out the surveys and experiments; J.Z. and W.X. contributed to the acquisition and interpretation of data; X.P. performed the statistical analysis; T.T. and Y.N. wrote the manuscript and prepared the tables; W.X. prepared the figures; G.S. supervised the work. All authors have read and agreed to the published version of the manuscript.

Funding: This research was funded by the National Natural Science Foundation of China (No.81872618), the sub-project of the National Key R\&D Program of China (No.2016YFC1305201), and Philosophy and Social Science Research Projects in Universities of Jiangsu Province (No.2020SJA2439).

Institutional Review Board Statement: This study was carried out in accordance with the Declaration of Helsinki and approved by the ethical committee of the China Center for Disease Control and Prevention. The ethical approval number was 201614. 
Informed Consent Statement: Informed consent was obtained from all participants involved in the study.

Data Availability Statement: The datasets used and/or analyzed during this study are available from the corresponding author on reasonable request.

Acknowledgments: The authors are grateful to the Chinese Center for Disease Control and Prevention for its support in this study's design and field work. We also appreciate the cooperation and compliance of all of the study's participants.

Conflicts of Interest: The authors declare that they have no conflicts of interest.

\section{References}

1. Millward, D.J. Nutrition, infection and stunting: The roles of deficiencies of individual nutrients and foods, and of inflammation, as determinants of reduced linear growth of children. Nutr. Res. Rev. 2017, 30, 50-72. [CrossRef] [PubMed]

2. Williams, A.M.; Suchdev, P.S. Assessing and Improving Childhood Nutrition and Growth Globally. Pediatric Clin. N. Am. 2017, 64, 755-768. [CrossRef] [PubMed]

3. Stephenson, J.; Heslehurst, N.; Hall, J.; Schoenaker, D.; Hutchinson, J.; Cade, J.E.; Poston, L.; Barrett, G.; Crozier, S.R.; Barker, M.; et al. Before the beginning: Nutrition and lifestyle in the preconception period and its importance for future health. Lancet 2018, 391, 1830-1841. [CrossRef]

4. Genovesi, S.; Parati, G. Cardiovascular Risk in Children: Focus on Pathophysiological Aspects. Int. J. Mol. Sci. 2020, 21, 6612. [CrossRef] [PubMed]

5. Sommer, A.; Twig, G. The Impact of Childhood and Adolescent Obesity on Cardiovascular Risk in Adulthood: A Systematic Review. Curr. Diabetes Rep. 2018, 18, 91. [CrossRef] [PubMed]

6. $\quad$ Bhutta, Z.A.; Berkley, J.A.; Bandsma, R.; Kerac, M.; Trehan, I.; Briend, A. Severe childhood malnutrition. Nat. Rev. Dis. Primers 2017, 3, 17067. [CrossRef]

7. Jahan, I.; Muhit, M.; Hardianto, D.; Laryea, F.; Amponsah, S.K.; Chhetri, A.B.; Smithers-Sheedy, H.; McIntyre, S.; Badawi, N.; Khandaker, G. Epidemiology of Malnutrition among Children with Cerebral Palsy in Low- and Middle-Income Countries: Findings from the Global LMIC CP Register. Nutrients 2021, 13, 3637. [CrossRef] [PubMed]

8. Perez-Escamilla, R.; Bermudez, O.; Buccini, G.S.; Kumanyika, S.; Lutter, C.K.; Monsivais, P.; Victora, C. Nutrition disparities and the global burden of malnutrition. BMJ 2018, 361, k2252. [CrossRef]

9. Song, P.; Wang, J.; Wei, W.; Chang, X.; Wang, M.; An, L. The Prevalence of Vitamin A Deficiency in Chinese Children: A Systematic Review and Bayesian Meta-Analysis. Nutrients 2017, 9, 1285. [CrossRef]

10. Huh, S.Y.; Gordon, C.M. Vitamin D deficiency in children and adolescents: Epidemiology, impact and treatment. Rev. Endocr. Metab. Disord. 2008, 9, 161-170. [CrossRef]

11. Li, H.; Xiao, J.; Liao, M.; Huang, G.; Zheng, J.; Wang, H.; Huang, Q.; Wang, A. Anemia prevalence, severity and associated factors among children aged 6-71 months in rural Hunan Province, China: A community-based cross-sectional study. BMC Public Health 2020, 20, 989. [CrossRef] [PubMed]

12. Iglesias Vázquez, L.; Valera, E.; Villalobos, M.; Tous, M.; Arija, V. Prevalence of Anemia in Children from Latin America and the Caribbean and Effectiveness of Nutritional Interventions: Systematic Review and Meta-Analysis. Nutrients 2019, 11, 183. [CrossRef] [PubMed]

13. Liu, X.; Piao, J.; Zhang, Y.; He, Y.; Li, W.; Yang, L.; Yang, X. Assessment of Zinc Status in School-Age Children from Rural Areas in China Nutrition and Health Survey 2002 and 2012. Biol. Trace Elem. Res. 2017, 178, 194-200. [CrossRef]

14. Dong, Y.; Jan, C.; Ma, Y.; Dong, B.; Zou, Z.; Yang, Y.; Xu, R.; Song, Y.; Ma, J.; Sawyer, S.M.; et al. Economic development and the nutritional status of Chinese school-aged children and adolescents from 1995 to 2014: An analysis of five successive national surveys. The lancet. Diabetes Endocrinol. 2019, 7, 288-299. [CrossRef]

15. Afshin, A.; Forouzanfar, M.H.; Reitsma, M.B.; Sur, P.; Estep, K.; Lee, A.; Marczak, L.; Mokdad, A.H.; Moradi-Lakeh, M.; GBD 2015 Obesity Collaborators; et al. Health Effects of Overweight and Obesity in 195 Countries over 25 Years. N. Engl. J. Med. 2017, 377, 13-27. [CrossRef]

16. DeBoer, M.D. Assessing and Managing the Metabolic Syndrome in Children and Adolescents. Nutrients 2019, 11, 1788. [CrossRef]

17. Lee, A.M.; Gurka, M.J.; DeBoer, M.D. Trends in Metabolic Syndrome Severity and Lifestyle Factors among Adolescents. Pediatrics 2016, 137, e20153177. [CrossRef]

18. Song, P.; Yu, J.; Chang, X.; Wang, M.; An, L. Prevalence and Correlates of Metabolic Syndrome in Chinese Children: The China Health and Nutrition Survey. Nutrients 2017, 9, 79. [CrossRef]

19. Li, N.; Zhang, S.; Li, W.; Wang, L.; Liu, H.; Li, W.; Zhang, T.; Liu, G.; Du, Y.; Leng, J. Prevalence of hyperuricemia and its related risk factors among preschool children from China. Sci. Rep. 2017, 7, 9448. [CrossRef]

20. Kumar, J.; Gupta, A.; Dev, K.; Kumar, S.; Kataria, D.; Gul, A.; Abbas, M.; Jamil, A.; Shahid, S.; Memon, S. Prevalence and Causes of Hyperuricemia in Children. Cureus 2021, 13, e15307. [CrossRef]

21. Ford, E.S.; Li, C.; Cook, S.; Choi, H.K. Serum concentrations of uric acid and the metabolic syndrome among US children and adolescents. Circulation 2007, 115, 2526-2532. [CrossRef] 
22. Mia, M.N.; Rahman, M.S.; Roy, P.K. Sociodemographic and geographical inequalities in under- and overnutrition among children and mothers in Bangladesh: A spatial modelling approach to a nationally representative survey. Public Health Nutr. 2018, 21, 2471-2481. [CrossRef]

23. Álvarez, C.; Guzmán-Guzmán, I.P.; Latorre-Román, P.Á.; Párraga-Montilla, J.; Palomino-Devia, C.; Reyes-Oyola, F.A.; ParedesArévalo, L.; Leal-Oyarzún, M.; Obando-Calderón, I.; Cresp-Barria, M.; et al. Association between the Sociodemographic Characteristics of Parents with Health-Related and Lifestyle Markers of Children in Three Different Spanish-Speaking Countries: An Inter-Continental Study at OECD Country Level. Nutrients 2021, 13, 2672. [CrossRef] [PubMed]

24. Zach, A.; Meyer, N.; Hendrowarsito, L.; Kolb, S.; Bolte, G.; Nennstiel-Ratzel, U.; Stilianakis, N.I.; Herr, C.; GME Study Group. Association of sociodemographic and environmental factors with the mental health status among preschool children-Results from a cross-sectional study in Bavaria, Germany. Int. J. Hyg. Environ. Health 2016, 219, 458-467. [CrossRef] [PubMed]

25. Yu, D.; Zhao, L.; Zhang, J.; Yang, Z.; Yang, L.; Huang, J.; Fang, H.; Guo, Q.; Xu, X.; Ju, L.; et al. China Nutrition and Health Surveys (1982-2017). China CDC Wkly. 2021, 3, 193-195. [CrossRef]

26. National Health and Family Planning Commission of People's Republic of China. Screening Standard for Malnutrition of School-Age Children and Adolescents; WS/T 456-2014; National Health and Family Planning Commission of People's Republic of China: Beijing, China, 2014.

27. National Health and Family Planning Commission of People's Republic of China. Screening for Overweight and Obesity among School-Age Children and Adolescents; WST 586-2018; National Health and Family Planning Commission of People's Republic of China: Beijing, China, 2018.

28. Cook, S.; Weitzman, M.; Auinger, P.; Nguyen, M.; Dietz, W.H. Prevalence of a metabolic syndrome phenotype in adolescents: Findings from the third National Health and Nutrition Examination Survey, 1988-1994. Arch. Pediatrics Adolesc. Med. 2003, 157, 821-827. [CrossRef] [PubMed]

29. Song, P.; Li, X.; Gasevic, D.; Flores, A.B.; Yu, Z. BMI, Waist Circumference Reference Values for Chinese School-Aged Children and Adolescents. Int. J. Environ. Res. Public Health 2016, 13, 589. [CrossRef] [PubMed]

30. Dong, Y.; Ma, J.; Song, Y.; Dong, B.; Wang, Z.; Yang, Z.; Wang, X.; Prochaska, J.J. National Blood Pressure Reference for Chinese Han Children and Adolescents Aged 7 to 17 Years. Hypertension 2017, 70, 897-906. [CrossRef]

31. Lee, J.M.; Okumura, M.J.; Davis, M.M.; Herman, W.H.; Gurney, J.G. Prevalence and determinants of insulin resistance among U.S. adolescents: A population-based study. Diabetes Care 2006, 29, 2427-2432. [CrossRef]

32. World Health Organization. Global Prevalence of Vitamin A Deficiency in Populations at Risk 1995-2005: WHO Global Database on Vitamin A Deficiency; WHO: Geneva, Switzerland, 2009.

33. Del Valle, H.B.; Yaktine, A.L.; Taylor, C.L. Dietary Reference Intakes for Calcium and Vitamin D; National Academies Press (US): Washington, DC, USA, 2011.

34. World Health Organization. Iron deficiency anaemia: Assessment, Prevention, and Control; A guide for Programme Managers; WHO: Geneva, Switzerland, 2001.

35. Liu, J.; Hanlon, A.; Ma, C.; Zhao, S.R.; Cao, S.; Compher, C. Low blood zinc, iron, and other sociodemographic factors associated with behavior problems in preschoolers. Nutrients 2014, 6, 530-545. [CrossRef] [PubMed]

36. Dong, Y.H.; Wang, Z.H.; Yang, Z.G.; Wang, X.J.; Chen, Y.J.; Zou, Z.Y.; Ma, J. Epidemic status and secular trends of malnutrition among children and adolescents aged 7-18 years from 2005 to 2014 in China. Beijing da xue xue bao. Yi xue ban J. Peking Univ. Health Sci. 2017, 49, 424-432.

37. Hu, X.; Jiang, H.; Wang, H.; Zhang, B.; Zhang, J.; Jia, X.; Wang, L.; Wang, Z.; Ding, G. Intraindividual Double Burden of Malnutrition in Chinese Children and Adolescents Aged 6-17 Years: Evidence from the China Health and Nutrition Survey 2015. Nutrients 2021, 13, 3097. [CrossRef] [PubMed]

38. Hales, C.M.; Fryar, C.D.; Carroll, M.D.; Freedman, D.S.; Ogden, C.L. Trends in Obesity and Severe Obesity Prevalence in US Youth and Adults by Sex and Age, 2007-2008 to 2015-2016. JAMA 2018, 319, 1723-1725. [CrossRef] [PubMed]

39. Yang, L.; Bovet, P.; Ma, C.; Zhao, M.; Liang, Y.; Xi, B. Prevalence of underweight and overweight among young adolescents aged 12-15 years in 58 low-income and middle-income countries. Pediatric Obes. 2019, 14, e12468. [CrossRef]

40. Wang, Y.; Beydoun, M.A.; Min, J.; Xue, H.; Kaminsky, L.A.; Cheskin, L.J. Has the prevalence of overweight, obesity and central obesity levelled off in the United States? Trends, patterns, disparities, and future projections for the obesity epidemic. Int. J. Epidemiol. 2020, 49, 810-823. [CrossRef]

41. Zhu, Y.; Pandya, B.J.; Choi, H.K. Prevalence of gout and hyperuricemia in the US general population: The National Health and Nutrition Examination Survey 2007-2008. Arthritis Rheum. 2011, 63, 3136-3141. [CrossRef] [PubMed]

42. Wang, R.; Zhang, H.; Hu, Y.C.; Chen, J.; Yang, Z.; Zhao, L.; Yang, L. Serum Vitamin A Nutritional Status of Children and Adolescents Aged 6-17 Years-China, 2016-2017. China CDC Wkly. 2021, 3, 189-192. [CrossRef] [PubMed]

43. Wang, S.; Shen, G.; Jiang, S.; Xu, H.; Li, M.; Wang, Z.; Zhang, S.; Yu, Y. Nutrient Status of Vitamin D among Chinese Children. Nutrients 2017, 9, 319. [CrossRef]

44. Wang, L.L.; Wang, H.Y.; Wen, H.K.; Tao, H.Q.; Zhao, X.W. Vitamin D status among infants, children, and adolescents in southeastern China. J. Zhejiang Univ. Sci. B 2016, 17, 545-552. [CrossRef] [PubMed]

45. Wu, J.; Hu, Y.; Li, M.; Chen, J.; Mao, D.; Li, W.; Wang, R.; Yang, Y.; Piao, J.; Yang, L.; et al. Prevalence of Anemia in Chinese Children and Adolescents and Its Associated Factors. Int. J. Environ. Res. Public Health 2019, 16, 1416. [CrossRef] [PubMed] 
46. Xu, H.; Li, Y.; Liu, A.; Zhang, Q.; Hu, X.; Fang, H.; Li, T.; Guo, H.; Li, Y.; Xu, G.; et al. Prevalence of the metabolic syndrome among children from six cities of China. BMC Public Health 2012, 12, 13. [CrossRef] [PubMed]

47. Dai, C.; Wang, C.; Xia, F.; Liu, Z.; Mo, Y.; Shan, X.; Zhou, Y. Age and Gender-Specific Reference Intervals for Uric Acid Level in Children Aged 5-14 Years in Southeast Zhejiang Province of China: Hyperuricemia in Children May Need Redefinition. Front. Pediatrics 2021, 9, 560720. [CrossRef] [PubMed]

48. Yang, C.; Chen, J.; Liu, Z.; Yun, C.; Li, Y.; Piao, J.; Yang, X. Association of Vitamin A Status with Overnutrition in Children and Adolescents. Int. J. Environ. Res. Public Health 2015, 12, 15531-15539. [CrossRef]

49. Tanumihardjo, S.A. Vitamin A: Biomarkers of nutrition for development. Am. J. Clin. Nutr. 2011, 94, 658S-665S. [CrossRef]

50. Zhong, M.; Kawaguchi, R.; Kassai, M.; Sun, H. Retina, retinol, retinal and the natural history of vitamin A as a light sensor. Nutrients 2012, 4, 2069-2096. [CrossRef] [PubMed]

51. Brett, N.R.; Lavery, P.; Agellon, S.; Vanstone, C.A.; Goruk, S.; Field, C.J.; Weiler, H.A. Vitamin D Status and Immune Health Outcomes in a Cross-Sectional Study and a Randomized Trial of Healthy Young Children. Nutrients 2018, 10, 680. [CrossRef] [PubMed]

52. Wimalawansa, S.J. Associations of vitamin D with insulin resistance, obesity, type 2 diabetes, and metabolic syndrome. J. Steroid Biochem. Mol. Biol. 2018, 175, 177-189. [CrossRef] [PubMed]

53. Kelishadi, R.; Farajzadegan, Z.; Bahreynian, M. Association between vitamin D status and lipid profile in children and adolescents: A systematic review and meta-analysis. Int. J. Food Sci. Nutr. 2014, 65, 404-410. [CrossRef]

54. Zittermann, A.; Gummert, J.F.; Börgermann, J. The role of vitamin D in dyslipidemia and cardiovascular disease. Curr. Pharm. Des. 2011, 17, 933-942. [CrossRef] [PubMed]

55. Yang, C.; Mao, M.; Ping, L.; Yu, D. Prevalence of vitamin D deficiency and insufficiency among 460,537 children in 825 hospitals from 18 provinces in mainland China. Medicine 2020, 99, e22463. [CrossRef]

56. Li, H.; Huang, T.; Xiao, P.; Zhao, X.; Liu, J.; Cheng, H.; Dong, H.; Morris, H.A.; Mi, J.; China Child and Adolescent Cardiovascular Health $(\mathrm{CCACH})$ Collaboration Group. Widespread vitamin D deficiency and its sex-specific association with adiposity in Chinese children and adolescents. Nutrition 2020, 71, 110646. [CrossRef]

57. Borrud, L.G.; Flegal, K.M.; Looker, A.C.; Everhart, J.E.; Harris, T.B.; Shepherd, J.A. Body Composition Data for Individuals 8 Years of Age and Older: U.S. Population, 1999-2004; Vital and Health Statistics Series 11; Data from the National Health Survey; U.S. Department of Health and Human Services: Washington, DC, USA, 2010; pp. 1-87.

58. Hazell, T.J.; DeGuire, J.R.; Weiler, H.A. Vitamin D: An overview of its role in skeletal muscle physiology in children and adolescents. Nutr. Rev. 2012, 70, 520-533. [CrossRef]

59. Alfredsson, L.; Armstrong, B.K.; Butterfield, D.A.; Chowdhury, R.; de Gruijl, F.R.; Feelisch, M.; Garland, C.F.; Hart, P.H.; Hoel, D.G.; Jacobsen, R.; et al. Insufficient Sun Exposure Has Become a Real Public Health Problem. Int. J. Environ. Res. Public Health 2020, 17, 5014. [CrossRef]

60. Wong, A.Y.; Chan, E.W.; Chui, C.S.; Sutcliffe, A.G.; Wong, I.C. The phenomenon of micronutrient deficiency among children in China: A systematic review of the literature. Public Health Nutr. 2014, 17, 2605-2618. [CrossRef]

61. Ma, G.; Li, Y.; Jin, Y.; Zhai, F.; Kok, F.J.; Yang, X. Phytate intake and molar ratios of phytate to zinc, iron and calcium in the diets of people in China. Eur. J. Clin. Nutr. 2007, 61, 368-374. [CrossRef]

62. Zhang, N.; Bécares, L.; Chandola, T. Patterns and Determinants of Double-Burden of Malnutrition among Rural Children: Evidence from China. PLoS ONE 2016, 11, e0158119. [CrossRef]

63. Ning, M.; Chang, H.H. Migration decisions of parents and the nutrition intakes of children left at home in rural China. Agric. Econ. 2013, 59, 467-477. [CrossRef]

64. Yang, W.; Li, X.; Li, Y.; Zhang, S.; Liu, L.; Wang, X.; Li, W. Anemia, malnutrition and their correlations with socio-demographic characteristics and feeding practices among infants aged 0-18 months in rural areas of Shaanxi province in northwestern China: A cross-sectional study. BMC Public Health 2012, 12, 1127. [CrossRef] [PubMed]

65. Jiang, Y.; Su, X.; Wang, C.; Zhang, L.; Zhang, X.; Wang, L.; Cui, Y. Prevalence and risk factors for stunting and severe stunting among children under three years old in mid-western rural areas of China. Child Care Health Dev. 2015, 41, 45-51. [CrossRef]

66. Smith, J.D.; Fu, E.; Kobayashi, M.A. Prevention and Management of Childhood Obesity and Its Psychological and Health Comorbidities. Annu. Rev. Clin. Psychol. 2020, 16, 351-378. [CrossRef] [PubMed]

67. Chen, P.; Wang, D.; Shen, H.; Yu, L.; Gao, Q.; Mao, L.; Jiang, F.; Luo, Y.; Xie, M.; Zhang, Y.; et al. Physical activity and health in Chinese children and adolescents: Expert consensus statement (2020). Br. J. Sports Med. 2020, 54, 1321-1331. [CrossRef] [PubMed]

68. Chen, J.L.; Esquivel, J.H.; Guo, J.; Chesla, C.A.; Tang, S. Risk factors for obesity in preschool-aged children in China. Int. Nurs. Rev. 2018, 65, 217-224. [CrossRef] [PubMed]

69. Gan, X.; Xu, W.; Yu, K. Economic Growth and Weight of Children and Adolescents in Urban Areas: A Panel Data Analysis on Twenty-Seven Provinces in China, 1985-2014. Child. Obes. 2020, 16, 86-93. [CrossRef] [PubMed] 